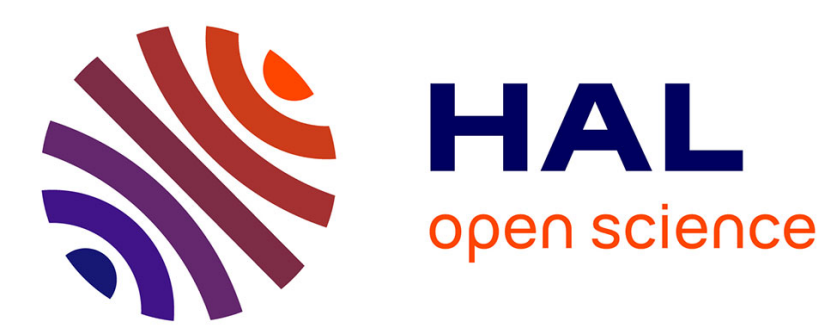

\title{
Informer et former sur l'open access et l'open data les chercheurs et les professionnels de l'IST
}

Chantal Salson, Hanka Hensens, Annabelle Filatre

\section{To cite this version:}

Chantal Salson, Hanka Hensens, Annabelle Filatre. Informer et former sur l'open access et l'open data les chercheurs et les professionnels de l'IST . Arabesques, 2016, 81, pp.18-19. hal-01689700

\section{HAL Id: hal-01689700 \\ https://hal.science/hal-01689700}

Submitted on 22 Jan 2018

HAL is a multi-disciplinary open access archive for the deposit and dissemination of scientific research documents, whether they are published or not. The documents may come from teaching and research institutions in France or abroad, or from public or private research centers.
L'archive ouverte pluridisciplinaire HAL, est destinée au dépôt et à la diffusion de documents scientifiques de niveau recherche, publiés ou non, émanant des établissements d'enseignement et de recherche français ou étrangers, des laboratoires publics ou privés. 


\section{Informer et former sur l'open access les professionnels de I'IST, les décideurs, les chercheurs.}

Compte-rendu des actions de formation sur l'open access, l'open data et l'open science financées par le projet Foster' ${ }^{1}$ à Agropolis $^{2}$, communauté scientifique de Montpellier et ses environs spécialisée en agriculture, alimentation, biodiversité et environnement.

\section{L'Open Access à Agropolis}

2700 scientifiques travaillent à Agropolis. En 2013, $16 \%$ de leurs publications référencées dans le Web of Science (Wos) étaient publiées dans des revues en libre accès ${ }^{3}$.

Les pratiques de dépôt de publications dans les archives institutionnelles diffèrent selon les instituts. Aucune institution n'a instauré de politique de dépôt obligatoire, mais une culture de partage existe, notamment dans la base Horizon de l'Institut de recherche pour le développement (IRD) et la base Open MeditAgri de I'Institut agronomique méditerranéen de Montpellier (CIHEAM-IAMM) ${ }^{4}$. En 2013, le dépôt de publications en libre accès est de 65\% pour le CIHEAM-IAMM, 54\% pour le centre de Montpellier de l'Institut de recherche en sciences et technologies pour l'environnement et l'agriculture (IRSTEA), 42\% pour l'IRD, 36\% pour le Cirad et la collection HAL Agropolis ${ }^{5}$, qui rassemble 4402 références de documents publiés par les 70 unités de recherche d'Agropolis, n'offre le libre accès que pour $47 \%$ d'entre eux.

L'enquête en ligne ${ }^{6}$ conduite pour recueillir les besoins et usages des scientifiques d'Agropolis en matière de données de la recherche, et les sensibiliser, montre que les bonnes pratiques de gestion et de dépôt de données sont mal connues et que les besoins d'accompagnement sont grands. Même si 1/3 des 174 personnes interrogées utilisent déjà archives ouvertes ou entrepôts de données, la marge de progression reste importante...

Suivant l'exemple de I'Université Montpellier 2 qui avait bénéficié d'un financement FOSTER en 2014, un groupe interinstitutionnel de documentalistes d'Agropolis ${ }^{7}$, a donc répondu à l'appel à projet FOSTER 2015 en proposant un programme de formation original et fédérateur, qui a été accepté ${ }^{8}$.

Les actions de formation projetées ciblaient 3 publics: les documentalistes, les managers de la recherche, et les scientifiques. Elles se sont déroulées tout au long de l'année 2015, notamment durant l'Open access week'.

\section{Former les formateurs}

La première série d'actions réalisée cherchait à renforcer et homogénéiser les connaissances des professionnels de l'Information scientifique et technique sur l'open access ${ }^{10}$ et l'open data ${ }^{11}$, pour

\footnotetext{
${ }^{1}$ FOSTER : Facilitate Open Science Training for European Research : projet européen FP7 de soutien d'actions de formation à l'open access.

${ }^{2}$ http://www.agropolis.fr

${ }^{3}$ Source : Etude bibliométrique de Dominique Fournier, Inra et Chantal Salson, Agropolis International sur les publications du pôle Agriculture Environnement Biodiversité Agropolis, de 2008 à 2013

${ }^{4}$ Autres entrepôts des instituts membres d'Agropolis : Prodlnra pour l'Inra, Agritrop pour le Cirad, CemOA pour l'Irstea

${ }^{5}$ https://hal.archives-ouvertes.fr/AGROPOLIS

${ }^{6}$ http://www.ist.agropolis.fr/les-formations/pour-les-etudiants-enseignants-et-chercheurs/item/open-access-et-open-data-pourla-communaute-scientifique-agropolis-projet-europeen-foster

${ }^{7}$ Documentalistes impliquées dans le groupe FOSTER/Agropolis : Cécile Adamolle (CIHEAM-IAM.M), Cécile Boussou (Cirad), Yannick Brohard (CNRS, UMR AMAP, réseau Doccitanist), Annabelle Filatre (AgroParisTech), Dominique Fournier (Inra), Hanka Hensens (IRD), Isabelle Nault (Irstea), Marie Nikichine (UM), Chantal Salson (Agropolis International), Christine Silvy (Inra, CBGP)

${ }^{8}$ En 2015, 80 projets ont été soumis, 25 ont été retenus en Europe, dont 2 en France: ceux de l'Université Paris Descartes et d'Agropolis International.

${ }^{9}$ Semaine du libre accès, évènement international annuel organisé depuis 2007.

${ }^{10}$ De l'Open Access à l'Open Data : Enjeux et perspectives / C. Silvy (INRA CBGP), le 5/3/2015: 13 assistants
} 
qu'ils puissent ensuite informer et former leurs usagers. Ces formations théoriques ont été complétées par deux ateliers pratiques de formation ${ }^{12}$ à la réalisation de screencasts, petites vidéos d'autoformation, qui ont permis de créer une chaîne YouTube Agropolis-Foster sur l'open access ${ }^{13}$. 35 professionnels de l'IST ont assisté à une ou plusieurs de ces trois formations.

\section{Sensibiliser les décideurs}

Plusieurs sessions d'information ont été organisées auprès des managers de la recherche (directeurs de laboratoires, directeurs et présidents d'instituts de recherche et d'enseignement supérieur), en pointant les préconisations de la Commission européenne dans le programme Horizon $2020^{14}$, (dépôt des publications, pour tous, et des données de la recherche, pour les projets pilotes), et le projet de loi pour une République numérique (et son article 9 sur la mise en accès libre des publications issues des travaux de recherche financés par des fonds publics). Les réactions ont porté sur l'évolution des modes de publication et de diffusion des résultats de la recherche : nouveaux modèles de revues, attachement à la validation des publications par les pairs, rigidité du système d'évaluation de la recherche et dévoiement du mouvement du libre accès, qui de libertaire à l'origine, tend à devenir libéral.

\section{Toucher les scientifiques}

Le public cible des ingénieurs, chercheurs, enseignants et étudiants était visé par : une exposition itinérante de posters, des fiches pratiques, des ateliers de «libération» de publications, une présentation des licences ouvertes pour les bases de données par une juriste du Cirad ${ }^{15}$, et une conférence organisée à la fin de l'Open access week.

Les 10 posters de l'exposition «L'open access ou comment libérer la production scientifique » ont été réalisés avec le groupe Open access Montpellier. Plusieurs jeux ont été imprimés et ont tourné dans 15 centres de documentation, hall d'instituts, cafétérias,... durant 7 semaines. Des visites guidées conviviales ont été organisées, et l'exposition virtuelle ${ }^{16}$ a rencontré un grand succès chez les scientifiques isolés.

La bibliothèque interuniversitaire de Montpellier a organisé un "grand défi » pour libérer les publications des chercheurs, qui a été décliné à l'IRD et à l'Inra, en s'appuyant notamment sur l'outil Dissem.in ${ }^{17}$, présenté à Montpellier par un de ses auteurs dans le cadre du projet. Les publications déjà libres d'accès et celles qui seraient libérables par dépôt dans une archive ouverte, ont ainsi été pointées, sur les listes de publications de chercheurs volontaires. Cette opération a entrainé de fructueuses discussions avec les auteurs, et le dépôt de près de 1000 documents à l'IRD et à l'INRA. 7 fiches pratiques ${ }^{18}$ sur l'open access, l'open data et la recherche ont été réalisées, mises en ligne et imprimées pour diffusion lors des diverses actions, d'autres ont été mises à jour.

\section{Une conférence co-organisée avec les chercheurs ${ }^{19}$}

La demi-journée d'information «open access et open data " comprenait deux présentations et deux tables-rondes.

Les professionnels de l'IST ont utilisé leurs relations de proximité avec les scientifiques pour les impliquer dans l'organisation de tables-rondes, leur permettant de témoigner de leurs pratiques.

110 personnes (dont 18 à distance), professionnels de l'information et scientifiques mêlés, ont écouté les retours d'expérience de chercheurs, d'ingénieurs, de documentalistes ou d'éditeurs, sur des pratiques, des politiques, enquêtes ou actions menées pour comprendre, promouvoir et accompagner

\footnotetext{
${ }^{11}$ Open Science : Gestion et partage des données de la recherche/Esther Dzale et Dominique L'Hostis (Pôle Open Data, Inra), le $1 / 4 / 2015: 27$ assistants

${ }^{12}$ Les $2 / 06$ et 17/07/2015, formatrices : Annabelle Filatre (AgroParisTech) et Hanka Hensens (IRD)

${ }^{13}$ https://www.youtube.com/channel/UCcQYqL3Jsgad5k7m40ILV Q

${ }^{14}$ Horizon 2020 est le plus grand programme de recherche et d'innovation de l'UE jamais créé : 80 milliards d' $€$ de financement sont disponibles sur 7 ans (2014-2020)

${ }^{15}$ Le 19/09/15 : 40 asistants

${ }^{16} \mathrm{http}: / /$ oam.biu-montpellier.fr/?page_id=735

${ }^{17}$ Dissemin est un service gratuit en version béta développé par des étudiants de l'Ecole Normale Supérieure de Paris : http://dissem.in/

${ }^{18}$ Fiches pratiques sur : http://ist.agropolis.fr/les-formations/tutoriels

${ }^{19}$ Programme et présentations sur : http://www.ist.agropolis.fr/fosteropenscience
} 
l'accès libre. Le débat entre les intervenants et avec la salle a été très riche : il semblerait que ce type de séminaire, court et participatif est plus apprécié qu'une conférence classique.

Le projet européen FOSTER a donné un cadre et un soutien financier pour la publication des différents supports d'information et les déplacements des intervenants des formations et de la conférence.

Il a permis la mobilisation des énergies autour d'actions innovantes mutualisées, essaimant dans les différentes structures membres d'Agropolis. Le groupe de travail open access constitué en 2015 poursuivra ses activités dans les années à venir.

Grâce à ces multiples actions, les professionnels de l'information d'Agropolis sont reconnus comme experts de l'open science, à même d'apporter un regard critique sur l'évolution de la diffusion des résultats de la science.

Chantal Salson Chargée de mission Information scientifique et technique à Agropolis International salson@agropolis.fr

Hanka Hensens Documentaliste au centre IRD de Montpellier hanka.hensens@ird.fr

Annabelle Filatre Documentaliste au centre AgroParisTech de Montpellier annabelle.filatre@agroparistech.fr 